\title{
Comparative Performance Analysis of Public Sector Sponsored and Private Sector Sponsored Mutual Funds in India
}

\author{
Anupam Thakuria \\ Associate Professor, Dept. of Economics, North Gauhati College, Guwahati (Assam) \\ Shikha Kashyap
}

Associate professor, Dept. of English, North Gauhati College, Guwahati (Assam)

\begin{abstract}
The concept of mutual funds in India dates back to the year 1963. The era between 1963 and 1987 marked the existence of only one mutual fund Company in India, namely the Unit Trust of India (UTI), with Rs. 67 billion assets under management (AUM). Few other mutual fund companies entered the mutual fund market later on. The private sector funds started penetrating the fund families during 1993. Kothari Pioneer was the first private sector mutual fund company in India which has now merged with Franklin Templeton. By the end of 1993, the total AUM of the industry was Rs. 470.04 billion. Just after ten years with private sector penetration, the total assets rose up to Rs. 1218. 05 billion and till 2004, it reached the height of 1540 billion. The total AUM of the mutual fund industry has risen up to 14000 billion in April, 2016. Today there are 43 mutual funds in India offering a number of schemes suited to the needs of different type of customers. It has been noticed that the private sector mutual funds have been taking more risks and have also been able to gain higher returns on an average. This paper tries to highlight the comparative performance of public and private sector mutual funds and also throw light on the scope of the existing potential of the fund market in the face of traditional risk aversion of the investors and huge rise in financial assets.
\end{abstract}

Keywords: Mutual Fund, Public sector, Private sector, Performance

\section{Introduction}

Mutual funds are pooled investments which enable investors to gain access to well diversified portfolios of securities. The amounts collected are professionally managed and invested in capital market instruments such as shares, debentures and other securities. It's one of the most suitable investment options as it offers an opportunity to invest in a diversified, professionally managed basket of securities at a relatively low cost and also provides liquidity to the investor as funds can be traded between the investor and the trust manager. In this sense, a mutual fund acts as a pure intermediary which performs the function of buying and selling securities on behalf of its Unit holders.

In India, the journey of mutual fund industry begins with the formation of Unit Trust of India (UTI) in the year 1963. There was the period of complete monopoly enjoyed by the UTI during 1963-1987. This actually marked the 1 st phase of the Indian Mutual Fund. The $2^{\text {nd }}$ phase is said to have lasted the period 1987-1993 when the Government of India allowed public sector banks and financial institutions to set up mutual funds. Various public sector players started entering the market during the later part of 1980s. In November 1987, SBI Mutual Fund from the State Bank of India became the first non-UTI mutual fund in India. This was followed by Canbank Mutual Fund, LIC Mutual Fund, Indian Bank Mutual Fund, Bank of India Mutual Fund, GIC Mutual Fund and PNB Mutual Fund. The $3^{\text {rd }}$ phase started with the entry of private sector and foreign funds during the period 1993-2003. During 1993, the private sector mutual funds started penetrating the market. Kothari Pioneer was the first private sector mutual fund company in India which has now merged with Franklin Templeton. Mutual Fund Regulations came into existence in 1993 with re-registering all mutual funds except UTI. In the beginning, the assets under management (AUM) of the UTI were a meager Rs. 67 billion. By the end of 1993, the total AUM of the fund industry was Rs. 470. 04 billion. By 1994-95, about 11 private sector funds had launched their schemes. However, UTI remained the leader with about $80 \%$ market share. The permission given to private sector funds including foreign fund 
management companies, which mostly entered the market through joint ventures with Indian promoters, provided a wide range of choice to investors and more competition in the industry. Private funds introduced innovative products, investment techniques and investor-servicing technology. SEBI (Mutual Funds) Regulations, 1996 was introduced by The Securities and Exchange Board of India (SEBI) that set uniform standards for all mutual funds in India. After 1996, the mutual fund industry witnessed robust growth and had also been subjected to stricter regulation by the (SEBI). With the increase in the number of players operating in the industry, fund mobilisation also increased and investors started showing more interest in mutual funds. SEBI looked into the aspects of Investors' interests and the Government too came forward to offer various incentives to the investors in order to encourage them.

Now is the age of consolidation and growth which actually started with the year 2003 . This can very well be dubbed as the $4^{\text {th }}$ phase of the Indian mutual fund industry. In February 2003, the UTI Act was repealed and UTI was stripped of its Special legal status as a trust. Presently Unit Trust of India operates under the name of UTI Mutual Fund. Its past schemes are being gradually wound up. However, UTI Mutual Fund has been still been the largest player in the industry. Thereafter, the mutual fund industry has come to witness several mergers and acquisitions. The acquisition of schemes of Alliance Mutual Fund by Birla Sun Life, Sun F\&C Mutual Fund and PNB Mutual Fund by Principal Mutual Fund has been some of the prominent examples. During this period, some more international mutual fund players like Fidelity, Franklin Templeton Mutual Fund etc. have entered India. This is a continuing phase of growth of the industry through consolidation and entry of new international and private sector players.

\section{Growth Potential of the mutual fund market}

The Indian Mutual Fund industry has witnessed a rapid growth in recent years. This growth is a combined result of a number of factors including infrastructural development, increase in personal financial assets and rise in foreign participation etc. It has become a preferred investment option compared to many other traditional investment avenues because of factors like growing risk appetite, rising income, increasing awareness and expectation of higher returns.

The Mutual fund industry in India has emerged as a dominant financial intermediary in Indian capital market. Just after ten years with private sector penetration, the total assets rose up to Rs. 1218. 05 billion and till 2004, it reached the height of 1540 billion. As of April 2006, the industry comprising of 33 Asset Management Companies managed financial assets of over Rs. 2000 billion (equivalent of US $\$ 45$ billion). This was the result of the continuous contributions by an estimated 20 million investors spread all over the country. The interesting point to note here is that though the volume of investment in mutual fund happens to be quite large, stocks and mutual funds only account for $4.95 \%$ of personal financial assets in India. Some experts have interpreted this as a kind of an indication of the huge potential in India for growth in investments by individuals into mutual funds and other risk assets. India has recently seen a rapid decline in the number of its extremely poor, along with an increase in its wealthy and middle-income segments. In India, the owners of mutual funds include not only the wealthy but also regular retail investors, and this should further broaden the market of potential mutual fund investors. Kamiyama, (2009). As on 31 March 2012, the average asset under management was Rs 66, 47, 920 million with a wide variety such as Open-Ended, Close-Ended, Interval, Growth, Income, Balanced, Equity Linked Savings Scheme (ELSS) and so on that caters to the investors' needs, risk tolerance and return expectations. The total AUM of the mutual fund industry has risen up to more than 14 trillion in April, 2016 (Economic Times, 6 May, 2016). Today there are 43 mutual funds in India offering a number of schemes suited to the needs of different type of customers.

The Assets under Management (AUM) have grown at a rapid pace at a CAGR of $35 \%$ over the 5 -year period from 31 March 2005 to 31 March 2009. Over the 10-year period from 1999 to 2009, the industry grew at 22\% CAGR. India has been amongst the fastest growing markets for mutual funds since 2004. During the period $2004-2008$, the Indian mutual fund industry grew at $29 \%$ CAGR as against the global average of $4 \%$. With this impressive growth, the ratio of AUM to GDP in India has also gradually increased from $6 \%$ in 2005 to $11 \%$ in 2009 . There has also been an increase in investment of the gross household financial savings into mutual funds from $1.2 \%$ in 2004 to $7.7 \%$ in 2008 . Gupta, 2011)

It has been observed that the saving pattern of Indian household sector is moving in favour of mutual funds. Traditionally, the asset portfolio of Indian household sector has been dominated by the time deposits and recurring deposits in the banks. Gradually, this trend has changed and there is more emphasis on investment in the mutual funds and the direct investment in the Securities market. Highly security-oriented Indian household sector is transformed into marginally risk-oriented sector and the risk-oriented segment of Indian Household sector has diverted the flow of their savings to the Stock markets through the medium of Mutual Funds. Raut, 2011). This interesting transformation of saving and investment decisions of Indian 
Household sector in recent years has become the cause of absolute growth of Mutual Fund Industry in India. Mutual Fund have mobilised resources largely from the household sector to the Capital market for transforming savings of the household sector into investment in the Capital market. There is positive trend of resource mobilisation by the Mutual Funds. Therefore, Mutual Funds have the potentials to promote rate of capital formation in Indian economy in the decade of 2010-11 to 202021.

\section{Unit Holding Pattern of all Mutual Funds}

Unit holding pattern of all Mutual Funds is more skewed towards individual household sector of Indian economy. On the other hand, unit holding pattern of corporate sector is the minimum. However, in spite of large unit holding by the individuals, there is less contribution to the total net assets by these individuals. On the contrary, in spite of minimum unit holding by the corporate sector, there is highest contribution to the total net assets by this sector. From the analysis of data on unit holding pattern of Private Sector Mutual Funds and Public Sector Sponsored Mutual Funds in India, the following observations are made:

1. Out of a total of 4.77 crore investors accounts in the mutual funds industry, 3.12 crore investors accounts i. e. $65.41 \%$ of the total investors accounts are in private sector mutual funds whereas the 1.65 crore investors accounts i. e. $34.59 \%$ are with the public sector sponsored mutual funds which ( includes UTI Mutual Fund.

2. However, the private sector mutual funds manage $77.97 \%$ of the net assets whereas the public sector sponsored mutual funds own only $22.03 \%$ of the assets.

Details of unit holding pattern of private sector and public sector sponsored mutual funds are given in the following tables:

Table 1: UNITHOLDING PATTERN OF PRIVATE SECTOR MFs (as on March 31, 2010)

\begin{tabular}{|l|l|l|l|l|}
\hline CATEGORY & $\begin{array}{l}\text { NUMBER OF } \\
\text { INVESTORS } \\
\text { ACCOUNTS }\end{array}$ & $\begin{array}{l}\text { \% TO TOTAL } \\
\text { INVESTORS } \\
\text { ACCOUNTS }\end{array}$ & $\begin{array}{l}\text { NET ASSETS } \\
\text { (RS. CRORE) }\end{array}$ & $\begin{array}{l}\text { \% TO } \\
\text { TOTAL NET } \\
\text { ASSETS }\end{array}$ \\
\hline Individuals & $30,041,859$ & $96.24 \%$ & $191,172.34$ & $39.74 \%$ \\
\hline NRIs & 787,791 & $2.52 \%$ & $24,703.76$ & $5.13 \%$ \\
\hline FIls & 211 & $0.00 \%$ & $6,204.35$ & $1.29 \%$ \\
\hline Corporates/Institutions/Others & 385,856 & $1.24 \%$ & $258,997.02$ & $53.84 \%$ \\
\hline TOTAL & $31,215,717$ & $100.00 \%$ & $481,077.47$ & $100.00 \%$ \\
\hline
\end{tabular}

Table2: UNITHOLDING PATTERN OF PUBLIC SECTOR SPONSORED MFS (INCLUDING UTI MF) (as on March 31, 2010)

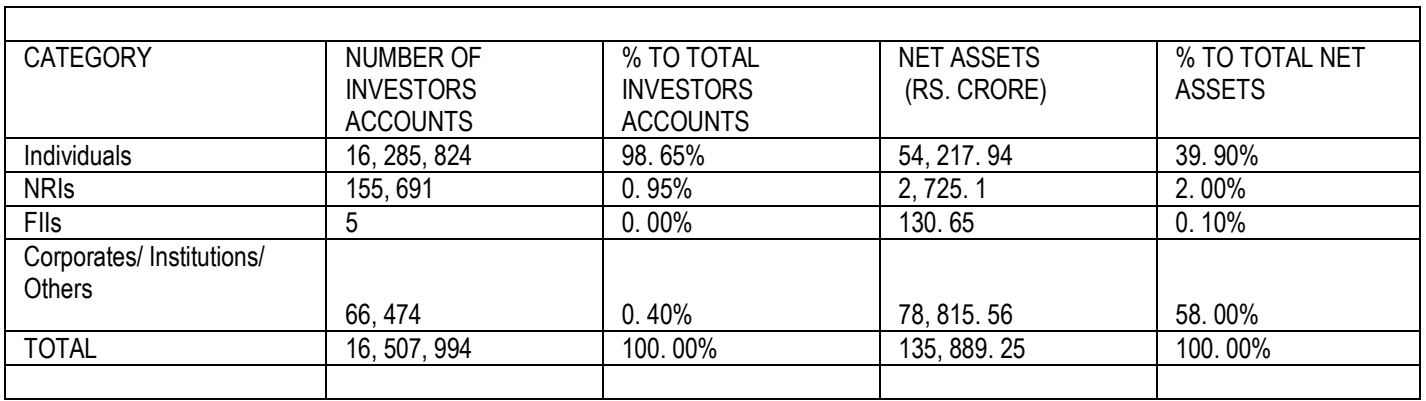

\section{Methodology and Analysis of Data}

This paper has tried to evaluate the growth of mutual funds during 2000-01 to 20015-16. It also aims to evaluate resource mobilisation and assets under management by mutual funds in India and to examine Unit holding pattern of Private and Public Sector mutual funds in India. The methodology adopted is to collect secondary data from various sources (mentioned 
in the References) and analyse this data with simple statistical tools such as Time Series Analysis and Correlation Regression Analysis.

First we have considered all the mutual funds existing in the market as on July, 2016 and have taken all the schemes offered by different funds. Various mutual funds have been offering open ended, close-ended, dividend option, growth option, direct and indirect schemes. We have taken the assets under management of all the AMCs and computed the one month, three months, one year and three lowest and highest performers based on the risk adjusted NAV figures. Then we have narrowed down our study to find out the performance of equity oriented schemes offered under direct and growth options.

\section{Performance Measurement}

Like all other studies on mutual funds, we too have measured the performance of mutual funds basically on the basis of three important models derived independently by Sharpe, Jensen and Treynor.

Sharpe Ratio gives the Fund return in excess of risk free return/ Standard deviation of Fund. Sharpe ratios are ideal for comparing funds that have a mixed asset class. The higher the Sharpe ratio, the better a fund's returns relative to the amount of risk taken.

Treynor ratio is the Fund return in excess of risk free return/ Beta of Fund. Treynor ratio indicates relative measure of market risk. The higher the Treynor ratio (higher will be the returns and lesser market risk of the fund.

Jensen's measure shows relative ratio between alpha and beta.

$\mathrm{R}$ - square measures the correlation of a fund's movement to that of an index. R-squared describes the level of association between the fund's volatility and market risk.

Standard Deviation allows us to evaluate the volatility of the fund. The standard deviation of a fund measures this risk by measuring the degree to which the fund fluctuates in relation to its mean return.

Beta is a fairly commonly used measure of risk. It basically indicates the level of volatility associated with the fund as compared to the benchmark. Beta $>1=$ high risk; Beta $=1=$ Average; Beta $<1=$ Low Risk

For understanding risk and return analysis, we have computed (Compounded Annual Growth Rate (CAGR) for 1 year, 2 year and 3 years, We have also calculated Standard deviation, (R- Square, Beta, Sharpe, (Treynor and Jensen measures. The results are classified on the basis of six categories of mutual funds like, i) Private AMCs, ii) Joint Venture predominantly Indian, iii) Joint venture foreign, iv) Joint venture Indian, v) IDBI and UTI and vi) DHFL. These classifications are based on the NAV India guidelines and computations are done by us from the data.

\section{Results and findings}

The tables appended in the later pages have given the results and calculations based on the data provided by NAV India. First they show the profiles of the mutual funds. The later tables show some of results based on certain set criteria.

\section{A. Performance in terms of periodic averages return analysis}

i) In terms of periodic averages return analysis based on risk adjusted net asset values for the period (June (to July 8, 2016, Birla Sun Life CEF - Global Agri Plan (G) under JV Indian category has been the lowest performer in the one month while Edelweiss Emerging Leaders Fund - Direct $(G)$ under Indian private category has emerged the best performer.

ii) In case of three months performance measures, for the period April 8 to July 8, 2016, Birla Sun Life Inter Equity - Plan A (D) under JV Indian category performed lowest and ICICI Pru Banking \& Financial Services - Direct (G) has emerged the best performer.

iii) In case of one year periodic returns for the period July 9, 2015 to July 8, 2016 Baroda Pioneer PSU Equity Fund - Plan $A(G)$ under the category JV Foreign performed lowest of all funds. Here Sundaram Rural India Fund - Direct (D) under Indian Private AMC has performed the best. 
iv) Again, in case of three year periodic average return analysis for the period July 9, 1013 to July 8, 2016, Taurus Ethical Fund - Direct (B) under Indian private category has performed the poorest of all. Here Joint Venture Indian AMC DSP BR Micro-Cap Fund - Direct $(G)$ has performed the best of all.

\section{B. Comparison of Schemes Performance based on Traditional Measures of Risk Analysis:}

\section{Standard Deviation}

Out of all the schemes, Escorts High Yield Equity Plan - Direct (D) under Indian Private (has got the highest S. D. with 26. 73 and Baroda Pioneer Bank \&Fin Serv Fund-Plan B-Dir (D-RI) under JV foreign has got the lowest S. D. at 1. 41.

\section{Sharpe ratio}

Two of the funds in the market, namely, Baroda Pioneer Bank\&Fin Serv Fund-Plan B-Dir (D-RI) and Tata Resources \& Energy Fund - Direct (D), both under Indian Private category have registered highest Sharpe ratio. On the other hand, Baroda Pioneer PSU Equity Fund - Plan B - Dir (D) under JV Foreign has shown lowest Sharpe ratio.

\section{Beta value}

Taurus Banking \& Financial Services - Direct (D) which is under Indian private AMC has shown highest Beta value during the study period; while two JV Indian funds namely, BOI AXA Mid Cap Equity \& Debt Fund - Direct (D) and SBI Shariha Equity Fund - Direct (D) have registered lowest Beta value

\section{Fama Ratio}

The scheme Tata Banking \& Financial Services Fund - Dir (D) under Indian Private AMC has shown highest Fama ratio during the period. The Joint venture foreign AMC Baroda Pioneer PSU Equity Fund - Plan B - Dir (D) has registered lowest Fama ratio.

Next we have calculated the alpha and beta values of different categories of mutual funds after computing the adjusted $R$ squares, standard errors by taking into account the intercept and coefficients of the regression equations. This has been done after computing the trend values risk-return analysis of different AMCs. Then we have found out the Sharpe ratio and Treynor ratio of different categories of funds. Both these ratios have indicated that Joint Venture foreign category of funds have outperformed the other categories in matters of getting rewards over risks of the portfolios. Again, the other methods of risk-return analysis based on standard deviations and adjusted NAV calculation clearly point to the fact that the private sector AMCs are generally inclined to taking more risks in their hunt for better returns. Even though many of them couldn't achieve better results over the long term horizon, some of them like, Reliance, Birla and Tata schemes have done well in risk-return analysis.

\section{References:}

[1] Barua, S. K. \& Verma, J. R. 1991, January - March). Mastershare: a bonanza for large investors. Vikalpa, 2934.

[2] Brown, S. J. \& Goetzman, W. N. 1997, June). Performance persistence. Journal of Finance, 679-698.

[3] Chang, E. C. \& (Lewellen W. 1984, January). Market timing and mutual fund investment performance. Journal of Business, 57-72.

[4] Cuthbertson, K. , Nitzsche, D. \& Sullivan, N. O. 2006). Mutual fund performance. Retrieved from http://ssrn. com/abstract $=955807$.

[5] Debbeeru, R. N. 2000). Investment styles and performance of equity mutual funds in (India. Available: http://ssrn. com/abstract

[6] Fama, E. F. \& French, K. R. 1993). Common risk factors in the returns on stocks and (bonds. Journal of financial Economics, 33 (4), (3-56. 
[7] Gupta, O. P. \& Sehgal, S. 1997). Investment performance of Mutual Funds - The Indian (experience. Mumbai: Tata McGraw Hill Publishing Company Ltd.

[8] Gupta, S. 2011, April). Indian mutual fund industry: Current state and future outlook. Indian Journal of Finance, $5(4), 119-121$

[9] Jain, P. K. 1982). Financial institution in India - A study of Unit Trust of India. New Delhi: (Triveni Publication.

[10] Jaydev, M. 1996). Mutual fund performance: An analysis of monthly returns. Finance India, 10 (1), 73-84.

[11] Kamiyama, T. 2009). India's mutual fund industry. Nomura Capital Market Review, 10 (4), 57-72.

[12] Khan, M. Y. \& (Jain, P. K. 2005). Financial management. New Delhi: Tata McGraw Hill.

[13] Mishra, B. 2001). A study of mutual funds in India. Un-published research paper under the aegis of Faculty of Management Studies, University of Delhi.

[14] Raut, O. 2011). Performance analysis of mutual funds in India, Retrieved from http://ssrn. com

[15] www. amfi. com

[16] www. sebi. com

[17] www. rbi. org.

Footnotes:

$A M C=$ (asset management company

AMFI = association of mutual funds in India

$\mathrm{AUM}=$ asset under management

$\mathrm{NRI}=$ non- resident Indian

$\mathrm{RBI}=$ Reserve Bank of India

S. E. = standard error

SEBI = Securities and Exchange Board of India

\section{Tables}

Table 3

\begin{tabular}{|c|c|c|c|c|c|c|c|}
\hline \multicolumn{8}{|c|}{$\begin{array}{l}\text { Asset Under Management of Various Categories of Asset Management Companies (Rs. } \\
\text { Crores) }\end{array}$} \\
\hline $\begin{array}{l}\text { Ye } \\
\text { ar }\end{array}$ & Foreign & $\begin{array}{l}\text { Indian } \\
\text { private }\end{array}$ & $\begin{array}{l}\mathrm{J} . \quad \mathrm{V} . \\
\text { Foreign }\end{array}$ & $\begin{array}{l}\text { J. V. } \\
\text { Indian }\end{array}$ & $\begin{array}{l}\text { J. V. } \\
\text { Predomina } \\
\text { ntly Indian }\end{array}$ & $\begin{array}{l}\text { IDBI \& } \\
\text { UTI }\end{array}$ & DHFL \\
\hline $\begin{array}{ll}200 \\
5\end{array}$ & $\begin{array}{l}4450 . \\
616 \\
\end{array}$ & $\begin{array}{l}2730 . \\
749\end{array}$ & $\begin{array}{l}3680 . \\
459\end{array}$ & $\begin{array}{l}8309 . \\
705\end{array}$ & 4810.273 & $\begin{array}{l}20617 . \\
37\end{array}$ & \\
\hline $\begin{array}{l}200 \\
6\end{array}$ & $\begin{array}{l}7854 . \\
583\end{array}$ & $\begin{array}{l}2655 . \\
902\end{array}$ & $\begin{array}{l}4925 . \\
021\end{array}$ & $\begin{array}{l}12660 . \\
95\end{array}$ & 14110.58 & $\begin{array}{l}25391 . \\
3\end{array}$ & \\
\hline $\begin{array}{l}200 \\
7\end{array}$ & 8984.3 & $\begin{array}{l}4188 . \\
96\end{array}$ & $\begin{array}{l}5788 . \\
07\end{array}$ & $\begin{array}{l}18345 \\
43\end{array}$ & 25567.11 & $\begin{array}{l}32262 . \\
93\end{array}$ & \\
\hline $\begin{array}{l}200 \\
8\end{array}$ & $\begin{array}{l}6296 . \\
61\end{array}$ & 3918.4 & $\begin{array}{l}5129 \\
32\end{array}$ & $\begin{array}{l}19296 . \\
07\end{array}$ & 34756.1 & $\begin{array}{l}37089 . \\
15\end{array}$ & \\
\hline $\begin{array}{l}200 \\
9\end{array}$ & $\begin{array}{l}7798 . \\
115\end{array}$ & $\begin{array}{l}5495 . \\
747\end{array}$ & $\begin{array}{l}4748 . \\
785 \\
\end{array}$ & $\begin{array}{l}32141 . \\
67 \\
\end{array}$ & 57121.91 & $\begin{array}{l}54326 . \\
71\end{array}$ & \\
\hline $\begin{array}{l}201 \\
0\end{array}$ & $\begin{array}{l}9905 . \\
864\end{array}$ & $\begin{array}{l}5068 . \\
532 \\
\end{array}$ & $\begin{array}{l}4649 . \\
067\end{array}$ & $\begin{array}{l}36393 \\
24\end{array}$ & 58301. 14 & $\begin{array}{l}31031 . \\
25 \\
\end{array}$ & \\
\hline $\begin{array}{l}201 \\
1 \\
\end{array}$ & $\begin{array}{l}9691 . \\
25\end{array}$ & $\begin{array}{l}5219 . \\
518 \\
\end{array}$ & $\begin{array}{l}3745 \\
823 \\
\end{array}$ & $\begin{array}{l}30509 . \\
76 \\
\end{array}$ & 42349.62 & $\begin{array}{l}28265 \\
74\end{array}$ & \\
\hline $\begin{array}{l}201 \\
2\end{array}$ & $\begin{array}{l}10733 . \\
92\end{array}$ & $\begin{array}{l}5276 . \\
049\end{array}$ & $\begin{array}{l}4905 . \\
421\end{array}$ & $\begin{array}{l}31390 . \\
35\end{array}$ & 36902.56 & $\begin{array}{l}28699 . \\
68\end{array}$ & $\begin{array}{l}68 . \\
71673 \\
\end{array}$ \\
\hline $\begin{array}{l}201 \\
3 \\
\end{array}$ & $\begin{array}{l}12251 . \\
3\end{array}$ & $\begin{array}{l}5719 . \\
969 \\
\end{array}$ & $\begin{array}{l}6641 . \\
914 \\
\end{array}$ & $\begin{array}{l}40450 . \\
12 \\
\end{array}$ & 46358.77 & $\begin{array}{l}33056 . \\
12 \\
\end{array}$ & $\begin{array}{l}93 . \\
91028 \\
\end{array}$ \\
\hline $\begin{array}{l}201 \\
4 \\
\end{array}$ & $\begin{array}{l}16256 . \\
01\end{array}$ & $\begin{array}{l}7718 . \\
309 \\
\end{array}$ & $\begin{array}{l}8025 \\
492 \\
\end{array}$ & $\begin{array}{l}55369 . \\
71\end{array}$ & 59608.48 & $\begin{array}{l}41099 . \\
69 \\
\end{array}$ & $\begin{array}{l}164 . \\
8091 \\
\end{array}$ \\
\hline $\begin{array}{l}201 \\
5\end{array}$ & $\begin{array}{l}21689 . \\
91\end{array}$ & $\begin{array}{l}10674 . \\
49\end{array}$ & $\begin{array}{l}8298 . \\
396\end{array}$ & $\begin{array}{l}71756 . \\
57\end{array}$ & 78981.64 & $\begin{array}{l}51524 . \\
9\end{array}$ & $\begin{array}{l}531 . \\
415 \\
\end{array}$ \\
\hline $\begin{array}{l}201 \\
6\end{array}$ & $\begin{array}{l}20836 . \\
76\end{array}$ & $\begin{array}{l}12478 . \\
3\end{array}$ & $\begin{array}{l}6823 \\
713\end{array}$ & $\begin{array}{l}80534 . \\
16\end{array}$ & 86656.15 & $\begin{array}{l}55986 . \\
3\end{array}$ & $\begin{array}{l}18018 . \\
45\end{array}$ \\
\hline
\end{tabular}

Table 4

\begin{tabular}{|c|c|c|c|c|c|c|c|}
\hline \multicolumn{8}{|c|}{ Market share based on Asset Under Management of Various Categories of Asset Management Companies } \\
\hline Year & Foreign & $\begin{array}{l}\text { Indian } \\
\text { Private }\end{array}$ & $\begin{array}{l}\text { Joint } \\
\text { Venture } \\
\text { Foreign }\end{array}$ & $\begin{array}{l}\text { Joint } \\
\text { Venture } \\
\text { Indian }\end{array}$ & $\begin{array}{l}\text { Joint Ventures- } \\
\text { Predominantly } \\
\text { Indian }\end{array}$ & $\begin{array}{l}\text { IDBI \& } \\
\text { UTI }\end{array}$ & DHFL \\
\hline 2005 & $\begin{array}{l}9 . \\
979145\end{array}$ & $\begin{array}{l}6 . \\
122869\end{array}$ & $\begin{array}{l}8 . \\
252303\end{array}$ & $\begin{array}{l}18 . \\
63197\end{array}$ & 10.78557 & $\begin{array}{l}46 . \\
22815\end{array}$ & 0 \\
\hline 2006 & $\begin{array}{l}11 . \\
61949\end{array}$ & $\begin{array}{l}3 . \\
928946\end{array}$ & $\begin{array}{l}7 . \\
285713\end{array}$ & $\begin{array}{l}18 . \\
72968\end{array}$ & 20.87415 & $\begin{array}{l}37 . \\
56202\end{array}$ & 0 \\
\hline 2007 & $\begin{array}{l}9 . \\
443559\end{array}$ & $\begin{array}{l}4 . \\
403091\end{array}$ & $\begin{array}{l}6 . \\
083944\end{array}$ & $\begin{array}{l}19 . \\
28321\end{array}$ & 26.87405 & $\begin{array}{l}33 . \\
91215\end{array}$ & 0 \\
\hline 2008 & $\begin{array}{l}5 . \\
913107\end{array}$ & $\begin{array}{l}3 . \\
679745\end{array}$ & $\begin{array}{l}4 . \\
816912\end{array}$ & $\begin{array}{l}18 . \\
12082\end{array}$ & 32.63923 & $\begin{array}{l}34 . \\
83019\end{array}$ & 0 \\
\hline 2009 & $\begin{array}{l}4 . \\
824583\end{array}$ & $\begin{array}{l}3 . \\
400141\end{array}$ & $\begin{array}{l}2 . \\
938006\end{array}$ & $\begin{array}{l}19 . \\
88559\end{array}$ & 35.34051 & $\begin{array}{l}33 . \\
61116\end{array}$ & 0 \\
\hline 2010 & $\begin{array}{l}6 . \\
8152222\end{array}$ & $\begin{array}{l}3 . \\
487144\end{array}$ & $\begin{array}{l}3 . \\
198552\end{array}$ & $\begin{array}{l}25 . \\
03851\end{array}$ & 40.11111 & $\begin{array}{l}21 . \\
34946\end{array}$ & 0 \\
\hline 2011 & $\begin{array}{l}8 . \\
090759\end{array}$ & $\begin{array}{l}4 . \\
357525\end{array}$ & $\begin{array}{l}3 . \\
127208\end{array}$ & $\begin{array}{l}25 . \\
47113\end{array}$ & 35.35566 & $\begin{array}{l}23 . \\
59771\end{array}$ & 0 \\
\hline 2012 & $\begin{array}{l}9 . \\
098339\end{array}$ & $\begin{array}{l}4 . \\
472111\end{array}$ & $\begin{array}{l}4 . \\
157958\end{array}$ & $\begin{array}{l}26 . \\
60725\end{array}$ & 31.27953 & $\begin{array}{l}24 . \\
32657\end{array}$ & $\begin{array}{l}0 . \\
058246\end{array}$ \\
\hline 2013 & 8.47418 & $\begin{array}{l}3 . \\
956482\end{array}$ & $\begin{array}{l}4 . \\
594188\end{array}$ & 27.9792 & 32.06619 & $\begin{array}{l}22 . \\
8648\end{array}$ & $\begin{array}{l}0 . \\
064957\end{array}$ \\
\hline 2014 & $\begin{array}{l}8 . \\
635675\end{array}$ & $\begin{array}{l}4 . \\
100195\end{array}$ & $\begin{array}{l}4 . \\
263379\end{array}$ & $\begin{array}{l}29 . \\
41403\end{array}$ & 31.66579 & $\begin{array}{l}21 . \\
83337\end{array}$ & $\begin{array}{l}0 . \\
087551\end{array}$ \\
\hline 2015 & $\begin{array}{l}8 . \\
909122\end{array}$ & $\begin{array}{l}4 . \\
384543\end{array}$ & $\begin{array}{l}3 . \\
408563\end{array}$ & $\begin{array}{l}29 . \\
47398\end{array}$ & 32. 44168 & $\begin{array}{l}21 . \\
16383\end{array}$ & $\begin{array}{l}0 . \\
218279\end{array}$ \\
\hline
\end{tabular}




\begin{tabular}{|l|l|l|}
\hline \multicolumn{3}{|l|}{ One Month Lowest Performers (June 8, 2016 to July 8, 2016) } \\
\hline $\begin{array}{l}\text { Asset Management } \\
\text { Category }\end{array}$ & Schemes & Return (adj nav) \\
\hline Joint Venture Indian & Birla Sun Life CEF - Global Agri Plan (G) & -5.4455 \\
\hline Joint Venture Indian & Birla Sun Life CEF - Global Agri Plan (D) & -5.4414 \\
\hline Joint Venture Indian & $\begin{array}{l}\text { Birla Sun Life CEF - Global Agri Plan - Direct } \\
\text { (G) }\end{array}$ & -5.4081 \\
\hline Joint Venture Indian & $\begin{array}{l}\text { Birla Sun Life CEF - Global Agri Plan - Direct } \\
\text { (D) }\end{array}$ & -5.4079 \\
\hline Joint Venture Indian & Birla Sun Life Inter Equity - Plan A (D) & -4.823 \\
\hline Joint Venture Indian & Birla Sun Life Inter Equity - Plan A (G) & -4.8228 \\
\hline Joint Venture Indian & Birla Sun Life Inter Equity - Plan A - Direct (G) & -4.7821 \\
\hline Joint Venture Indian & Birla Sun Life Inter Equity - Plan A - Direct (D) & -4.7817 \\
\hline Indian Private AMC & Tata Digital India Fund (D) & -4.6719 \\
\hline Indian Private AMC & Tata Digital India Fund (G) & -4.6719 \\
\hline
\end{tabular}

\begin{tabular}{|l|l|l|}
\hline \multicolumn{3}{|l|}{ One Month Highest Performers (June 8,2016 to July 8, 2016) } \\
\hline $\begin{array}{l}\text { Asset Management } \\
\text { Category }\end{array}$ & Schemes & Return (adj nav) \\
\hline Indian Private Company & $\begin{array}{l}\text { L\&T Emerging Businesses Fund - Direct } \\
\text { (D) }\end{array}$ & 8.74 \\
\hline Indian Private Company & Sundaram PSU Opportunities Fund (G) & 9.2435 \\
\hline Indian Private Company & Sundaram PSU Opportunities Fund (D) & 9.2437 \\
\hline Indian Private Company & $\begin{array}{l}\text { Sundaram PSU Opportunities Fund - } \\
\text { Direct (G) }\end{array}$ & 9.2479 \\
\hline Indian Private Company & $\begin{array}{l}\text { Sundaram PSU Opportunities Fund - } \\
\text { Direct (D) }\end{array}$ & 9.2483 \\
\hline Indian Private Company & Edelweiss Emerging Leaders Fund (G) & 10.4055 \\
\hline Indian Private Company & Edelweiss Emerging Leaders Fund (D) & 10.4521 \\
\hline Foreign AMC & GS PSU Bank BeES & 10.4755 \\
\hline Indian Private Company & $\begin{array}{l}\text { Edelweiss Emerging Leaders Fund - } \\
\text { Direct (D) }\end{array}$ & 10.5061 \\
\hline Indian Private Company & $\begin{array}{l}\text { Edelweiss Emerging Leaders Fund - } \\
\text { Direct (G) }\end{array}$ & 10.5137 \\
\hline
\end{tabular}

Table 7

\begin{tabular}{|l|l|l|}
\hline \multicolumn{3}{|l|}{ Three Month Lowest Performers (April 8, 2016 to July 8, 2016) } \\
\hline $\begin{array}{l}\text { Asset Management } \\
\text { Category }\end{array}$ & Schemes & Return (adj nav) \\
\hline Joint Venture Indian & Birla Sun Life Inter Equity - Plan A (D) & -4.4781 \\
\hline Joint Venture Indian & Birla Sun Life Inter Equity - Plan A (G) & -4.4777 \\
\hline & $\begin{array}{l}\text { Birla Sun Life Inter Equity - Plan A - Direct } \\
\text { (G) }\end{array}$ & -4.3552 \\
\hline Joint Venture Indian & $\begin{array}{l}\text { Birla Sun Life Inter Equity - Plan A - Direct } \\
\text { (D) }\end{array}$ & -4.3546 \\
\hline Joint Venture Indian & Tata Digital India Fund (D) & -1.8048 \\
\hline Indian Private AMC & Tata Digital India Fund (G) & -1.8048 \\
\hline Indian Private AMC & Tata Digital India Fund - Direct (D) & -1.4082 \\
\hline Indian Private AMC & Tata Digital India Fund - Direct (G) & -1.4082 \\
\hline Indian Private AMC & HDFC Arbitrage Fund - WP (Div-M) & 0.1109 \\
\hline Joint Venture Indian & HDFC Arbitrage Fund - WP - Direct (Div-M) & 0.1925 \\
\hline & Joint Venture Indian & \\
\hline
\end{tabular}

Table 9

\begin{tabular}{|l|l|l|}
\hline \multicolumn{3}{|l|}{ One Year Lowest Performers (July 9, 2015 to July 8, 2016) } \\
\hline $\begin{array}{l}\text { Asset Management } \\
\text { Category }\end{array}$ & $\begin{array}{l}\text { Return } \\
\text { nav) }\end{array}$ \\
\hline J. V. Foreign & $\begin{array}{l}\text { Baroda Pioneer PSU Equity Fund - Plan A } \\
\text { (G) }\end{array}$ & -18.0974 \\
\hline J. V. Foreign & $\begin{array}{l}\text { Baroda Pioneer PSU Equity Fund - Plan A } \\
\text { (D) }\end{array}$ & -18.0974 \\
\hline J. V. Foreign & $\begin{array}{l}\text { Baroda Pioneer PSU Equity Fund - Plan B - } \\
\text { Dir (G) }\end{array}$ & -17.5199 \\
\hline J. V. Foreign & $\begin{array}{l}\text { Baroda Pioneer PSU Equity Fund - Plan B - } \\
\text { Dir (D) }\end{array}$ & -17.5199 \\
\hline Foreign AMC & GS PSU Bank BeES & -16.8942 \\
\hline Joint Venture Indian & HDFC Large Cap Fund (D) & -12.8772 \\
\hline $\begin{array}{l}\text { J. V. Predominantly } \\
\text { (Indian }\end{array}$ & Reliance Capital Builder Fund II - Sr. B (G) & -12.1671 \\
\hline $\begin{array}{l}\text { J. V. Predominantly } \\
\text { (Indian }\end{array}$ & Reliance Capital Builder Fund II - Sr. B (D) & -12.1671 \\
\hline $\begin{array}{l}\text { J. V. Predominantly } \\
\text { (Indian }\end{array}$ & Reliance Japan Equity Fund (G) & -11.0699 \\
\hline $\begin{array}{l}\text { J. V. Predominantly } \\
\text { (Indian }\end{array}$ & Reliance Japan Equity Fund (D) & -11.0699 \\
\hline
\end{tabular}

Table 11
Table 8

\begin{tabular}{|l|l|l|}
\hline \multicolumn{2}{|l|}{ Three Month Highest Performers (April 8, 2016 to July 8, 2016) } \\
\hline $\begin{array}{l}\text { Asset Management } \\
\text { Category }\end{array}$ & $\begin{array}{l}\text { Return (adj } \\
\text { nav) }\end{array}$ \\
\hline Joint Venture Indian & $\begin{array}{l}\text { Birla Sun Life Banking \& Financial Services- } \\
\text { Dir (G) }\end{array}$ & 22.0994 \\
\hline Joint Venture Indian & $\begin{array}{l}\text { Birla Sun Life Banking \& Financial Services- } \\
\text { Dir (D) }\end{array}$ & 22.19 \\
\hline $\begin{array}{l}\text { J. V. Predominantly } \\
\text { Indian }\end{array}$ & Reliance Capital Builder Fund II - Sr. A (G) & 22.5056 \\
\hline $\begin{array}{l}\text { J. V. Predominantly } \\
\text { (Indian }\end{array}$ & Reliance Capital Builder Fund II - Sr. A (D) & 22.5056 \\
\hline $\begin{array}{l}\text { J. V. Predominantly } \\
\text { Indian }\end{array}$ & $\begin{array}{l}\text { Reliance Capital Builder Fund II - Sr. A - Dir } \\
\text { (G) }\end{array}$ & 22.6792 \\
\hline $\begin{array}{l}\text { J. V. Predominantly } \\
\text { (Indian }\end{array}$ & $\begin{array}{l}\text { Reliance Capital Builder Fund II - Sr. A - Dir } \\
\text { (D) }\end{array}$ & 22.6792 \\
\hline Joint Venture Indian & ICICI Pru Banking \& Financial Services (G) & 27.1429 \\
\hline Joint Venture Indian & ICICI Pru Banking \& Financial Services (D) & 27.1758 \\
\hline $\begin{array}{l}\text { Joint Venture Indian } \\
\text { Direct Pru Banking \& Financial Services - }\end{array}$ & 27.4882 \\
\hline $\begin{array}{l}\text { Joint Venture } \\
\text { Indian }\end{array}$ & $\begin{array}{l}\text { ICICI Pru Banking \& Financial Services - } \\
\text { Direct (G) }\end{array}$ & 27.5196 \\
\hline
\end{tabular}

Table 10

\begin{tabular}{|l|l|l|}
\hline \multicolumn{2}{|l|}{ One Year Highest Performers (July 9, 2015 to July 8, 2016) } \\
\hline $\begin{array}{l}\text { Asset } \\
\text { Category }\end{array}$ & Sanagemenes & Return (adj nav) \\
\hline Indian Private AMC & $\begin{array}{l}\text { Escorts High Yield Equity Plan - Direct } \\
\text { (B) }\end{array}$ & 16.849 \\
\hline Joint Venture Indian & DSP BR Micro-Cap Fund (D) & 16.9727 \\
\hline Joint Venture Indian & DSP BR Micro-Cap Fund (G) & 16.976 \\
\hline Joint Venture Indian & DSP BR Micro-Cap Fund - Direct (G) & 17.838 \\
\hline Joint Venture Indian & DSP BR Micro-Cap Fund - Direct (D) & 17.8399 \\
\hline Indian Private AMC & Sundaram Rural India Fund (G) & 18.3849 \\
\hline Indian Private AMC & Sundaram Rural India Fund (D) & 18.5857 \\
\hline Indian Private AMC & Sundaram Rural India Fund - Inst (D) & 18.7622 \\
\hline Indian Private AMC & Sundaram Rural India Fund - Direct (G) & 18.8715 \\
\hline Indian Private AMC & Sundaram Rural India Fund - Direct (D) & 19.0348 \\
\hline
\end{tabular}

Table 12 


\begin{tabular}{|l|l|l|}
\hline \multicolumn{4}{|l|}{ Three Year Lowest Performers (July 9, 2013 to July 8, 2016) } \\
\hline $\begin{array}{l}\text { Asset Management } \\
\text { Category }\end{array}$ & Schemes & $\begin{array}{l}\text { Return (adj } \\
\text { nav) }\end{array}$ \\
\hline Indian Private AMC & Taurus Ethical Fund - Direct (B) & -40.9836 \\
\hline J. V. Foreign & HSBC Emerging Markets Fund (D) & 5.8811 \\
\hline J. V. Foreign & HSBC Emerging Markets Fund (G) & 5.9331 \\
\hline J. V. Foreign & $\begin{array}{l}\text { HSBC Emerging Markets Fund - Direct } \\
\text { (D) }\end{array}$ & 8.1733 \\
\hline J. V. Foreign & $\begin{array}{l}\text { HSBC Emerging Markets Fund - Direct } \\
\text { (G) }\end{array}$ & 8.1891 \\
\hline Joint Venture Indian & HDFC Arbitrage Fund - WP (Div-M) & 8.3 \\
\hline Foreign AMC & GS PSU Bank BeES & 8.4194 \\
\hline Joint Venture Indian & Birla Sun Life Inter Equity - Plan A (G) & 9.173 \\
\hline Joint Venture Indian & Birla Sun Life Inter Equity - Plan A (D) & 9.217 \\
\hline Joint Venture Indian & $\begin{array}{l}\text { Birla Sun Life Inter Equity - Plan A - Direct } \\
\text { (G) }\end{array}$ & 10.8189 \\
\hline
\end{tabular}

\begin{tabular}{|c|c|c|}
\hline Asset Management Catego & Schemes & $\begin{array}{l}\begin{array}{l}\text { Return (adj } \\
\text { nav) }\end{array} \\
\end{array}$ \\
\hline $\begin{array}{l}\text { J. V. Predominantly } \\
\text { (Indian }\end{array}$ & Reliance Small Cap Fund $(G)$ & 202.6651 \\
\hline $\begin{array}{l}\text { J. V. Predominantly } \\
\text { (Indian }\end{array}$ & Reliance Small Cap Fund (B) & 202.6651 \\
\hline $\begin{array}{l}\text { J. V. Predominantly } \\
\text { (Indian }\end{array}$ & Reliance Small Cap Fund (D) & 202.6849 \\
\hline $\begin{array}{l}\text { J. V. Predominantly } \\
\text { (Indian }\end{array}$ & Reliance Small Cap Fund - Direct (B) & 210.5775 \\
\hline $\begin{array}{l}\text { J. V. Predominantly } \\
\text { (Indian }\end{array}$ & Reliance Small Cap Fund - Direct (G) & 210.5775 \\
\hline $\begin{array}{l}\text { J. V. Predominantly } \\
\text { (Indian }\end{array}$ & Reliance Small Cap Fund - Direct (D) & 210.962 \\
\hline Joint Venture Indian & DSP BR Micro-Cap Fund (D) & 219.4102 \\
\hline Joint Venture Indian & DSP BR Micro-Cap Fund (G) & 219.4203 \\
\hline Joint Venture Indian & DSP BR Micro-Cap Fund - Direct (D) & 225.9933 \\
\hline Joint Venture Indian & DSP BR Micro-Cap Fund - Direct (G) & 226.0427 \\
\hline
\end{tabular}

Comparison of Schemes Performance based on Traditional Measures of Risk Analysis: Standard Deviation, Sharpe Ratio, Beta, Fama Ratio

Table 13

\begin{tabular}{|l|l|l|}
\hline \multicolumn{3}{|l|}{ Schemes with Highest Standard Deviation } \\
\hline $\begin{array}{l}\text { Asset Management } \\
\text { Category }\end{array}$ & Schemes & SD \\
\hline J. V. Indian & HDFC Infrastructure Fund - Direct (D) & 2.00 \\
\hline J. V. Predominantly Indian & Reliance Banking Fund - Direct (D) & 2.03 \\
\hline UTI & UTI-Banking Sector Fund - Direct (D) & 2.15 \\
\hline Indian Private AMC & Taurus Banking \& Financial Services - Direct (D) & 2.22 \\
\hline Foreign AMC & Invesco India Banking Fund - Direct (D) & 2.23 \\
\hline J. V. Foreign & $\begin{array}{l}\text { Baroda Pioneer Bank\&Fin Serv Fund - Plan B- } \\
\text { Dir (D) }\end{array}$ & 2.29 \\
\hline Indian Private AMC & Sahara Banking \& Financial Services - Direct (D) & 2.30 \\
\hline J. V. Foreign & Principal Dividend Yield Fund - Direct (Div-H) & 3.19 \\
\hline Indian Private AMC & Peerless Equity Fund - Direct (Div-Q) & 3.74 \\
\hline Indian Private AMC & Escorts High Yield Equity Plan - Direct (D) & 26.73 \\
\hline
\end{tabular}

Table 15
Table 14

\begin{tabular}{|l|l|l|}
\hline \multicolumn{2}{|l|}{ Schemes with Lowest Standard Deviation } \\
\hline $\begin{array}{l}\text { Asset } \\
\text { Category }\end{array}$ & Management & Schemes \\
\hline J. V. Foreign & $\begin{array}{l}\text { Baroda Pioneer Bank\&Fin Serv Fund-Plan B-Dir } \\
\text { (D-RI) }\end{array}$ & 1.41 \\
\hline J. V. Indian & $\begin{array}{l}\text { BOI AXA Mid Cap Equity \& Debt Fund - Direct } \\
\text { (D) }\end{array}$ & 1.42 \\
\hline J. V. Indian & SBI Shariha Equity Fund - Direct (D) & 1.47 \\
\hline Indian Private AMC & Sundaram Equity Plus - Direct (D) & 1.49 \\
\hline UTI & UTI-Wealth Builder Fund - Direct (D) & 1.49 \\
\hline Indian Private AMC & Tata Ethical Fund - Direct (D) & 1.51 \\
\hline J.V. Indian & SBI Magnum Global Fund - Direct (D) & 1.52 \\
\hline Foreign AMC & Invesco India Dynamic Equity Fund - Direct (D) & 1.79 \\
\hline UTI & UTI-MNC Fund - Direct (D) & 1.93 \\
\hline Indian Private AMC & Peerless Midcap Fund - Direct (D) & 5.17 \\
\hline
\end{tabular}

Table 16 


\begin{tabular}{|l|l|l|}
\hline \multicolumn{2}{|l|}{ Schemes with Highest Sharpe Ratio } \\
\hline $\begin{array}{l}\text { Asset Management } \\
\text { Category }\end{array}$ & Highest Sharpe ratio & $\begin{array}{l}\text { Shapre } \\
\text { Ratio }\end{array}$ \\
\hline J. V. Indian & Birla Sun Life MNC Fund - Direct (D) & 0.10 \\
\hline UTI & $\begin{array}{l}\text { UTI-Transportation \& Logistics Fund - Direct } \\
\text { (D) }\end{array}$ & 0.11 \\
\hline Indian Private AMC & $\begin{array}{l}\text { Motilal Oswal Most Focused Multicap 35 Fund- } \\
\text { Dir (D) }\end{array}$ & 0.11 \\
\hline J. V. Indian & $\begin{array}{l}\text { ICICI Pru Exports and Other Services Fund - } \\
\text { Dir (D) }\end{array}$ & 0.11 \\
\hline Foreign AMC & $\begin{array}{l}\text { Franklin India Smaller Companies Fund - } \\
\text { Direct (D) }\end{array}$ & 0.11 \\
\hline IDBI & IDBI Diversified Equity Fund - Direct (D) & 0.11 \\
\hline Indian Private AMC & $\begin{array}{l}\text { Motilal Oswal Most Focused Midcap 30 Fund- } \\
\text { Dir (D) }\end{array}$ & 0.11 \\
\hline Indian Private AMC & $\begin{array}{l}\text { Tata Banking \& Financial Services Fund - Dir } \\
\text { (D) }\end{array}$ & 0.12 \\
\hline IDBI & IDBI Equity Advantage Fund - Direct (D) & 0.12 \\
\hline Indian Private AMC & Tata Resources \& Energy Fund - Direct (D) & 0.12 \\
\hline
\end{tabular}

\begin{tabular}{|c|c|c|}
\hline \multicolumn{3}{|c|}{ Schemes with Lowest Sharpe Ratio } \\
\hline $\begin{array}{l}\text { Asset } \\
\text { Category }\end{array}$ & Lowest Sharpe Ratio & $\begin{array}{l}\text { Sharpe } \\
\text { Ratio }\end{array}$ \\
\hline J. V. Foreign & $\begin{array}{l}\text { Baroda Pioneer PSU Equity Fund - Plan B - } \\
\text { Dir (D) }\end{array}$ & -0.04 \\
\hline Indian Private AMC & $\begin{array}{l}\text { Tata India Pharma \& Healthcare Fund - Direct } \\
\text { (D) }\end{array}$ & -0.04 \\
\hline J. V. Predominantly Indian & LIC MF Banking \& Fina Serv Fund - Dir (D) & -0.02 \\
\hline Indian Private AMC & JM Equity Fund - Direct (Div-H) & -0.01 \\
\hline J.V. Predominanty Indian & $\begin{array}{l}\text { Reliance Tax Saver (ELSS) Fund - Direct } \\
\text { (Div-A) }\end{array}$ & -0.01 \\
\hline Indian Private AMC & JM Equity Fund - Direct (Div-A) & -0.01 \\
\hline Indian Private AMC & JM Equity Fund - Direct (Div-M) & -0.01 \\
\hline Indian Private AMC & JM Equity Fund - Direct (Div-Q) & -0.01 \\
\hline J. V. Indian & $\begin{array}{l}\text { JPMorgan India Economic Resurgence Fund } \\
\text { - Dir (D) }\end{array}$ & -0.01 \\
\hline DHFL & $\begin{array}{l}\text { DHFL Pramerica Diversified Equity Fund - Dir } \\
\text { (D) }\end{array}$ & 0.00 \\
\hline
\end{tabular}

Table 17

\begin{tabular}{|l|l|l|}
\hline \multicolumn{2}{|l|}{ Schemes with Highest Beta } \\
\hline $\begin{array}{l}\text { Asset Management } \\
\text { Category }\end{array}$ & Schemes & Beta \\
\hline J. V. Indian & $\begin{array}{l}\text { Birla Sun Life Banking \& Financial Services-Dir } \\
\text { (D) }\end{array}$ & 1.16 \\
\hline Foreign AMC & Invesco India Banking Fund - Direct (D) & 1.16 \\
\hline Indian Private AMC & $\begin{array}{l}\text { Sahara Banking \& Financial Services - Direct } \\
\text { (D) }\end{array}$ & 1.18 \\
\hline Indian Private AMC & Sundaram Fin Serv Opportunities - Direct (D) & 1.19 \\
\hline Indian Private AMC & JM Core 11 Fund - Direct (D) & 1.20 \\
\hline J. V. Indian & HDFC Infrastructure Fund - Direct (D) & 1.20 \\
\hline J. V. Predominantly Indian & Reliance Banking Fund - Direct (D) & 1.22 \\
\hline UTI & UTI-Banking Sector Fund - Direct (D) & 1.25 \\
\hline J. V. Foreign & $\begin{array}{l}\text { Baroda Pioneer Bank\&Fin Serv Fund - Plan B- } \\
\text { Dir (D) }\end{array}$ & 1.26 \\
\hline Indian Private AMC & $\begin{array}{l}\text { Taurus Banking \& Financial Services - Direct } \\
\text { (D) }\end{array}$ & 1.28 \\
\hline
\end{tabular}

Table 18

\begin{tabular}{|l|l|l|}
\hline \multicolumn{3}{|l|}{ Schemes with Lowest Beta } \\
\hline $\begin{array}{l}\text { Asset Management } \\
\text { Category }\end{array}$ & Schemes & Beta \\
\hline J. V. Foreign & $\begin{array}{l}\text { Baroda Pioneer Bank\&Fin Serv Fund-Plan B- } \\
\text { Dir (D-RI) }\end{array}$ & 0.00 \\
\hline J. V. Indian & $\begin{array}{l}\text { BOI AXA Mid Cap Equity \& Debt Fund - Direct } \\
\text { (D) }\end{array}$ & 0.00 \\
\hline J. V. Indian & SBI Sharha Equity Fund - Direct (D) & 0.00 \\
\hline J. V. Indian & ICICI Pru Technology Fund - Direct (D) & 0.49 \\
\hline J. V. Predominantly Indian & Reliance Pharma Fund - Direct (D) & 0.52 \\
\hline J. V. Predominantly Indian & SBI Magnum Global Fund - Direct (D) & 0.53 \\
\hline Foreign AMC & Franklin Infotech Fund - Direct (D) & 0.53 \\
\hline UTI & UTI-Pharma \& Healthcare Fund - Direct (D) & 0.56 \\
\hline J. V. Indian & $\begin{array}{l}\text { ICICI Pru Exports and Other Services Fund - } \\
\text { Dir (D) }\end{array}$ & 0.56 \\
\hline Indian Private AMC & $\begin{array}{l}\text { Tata India Pharma \& Healthcare Fund - Direct } \\
\text { (D) }\end{array}$ & 0.57 \\
\hline
\end{tabular}

Table 19

\begin{tabular}{|l|l|l|}
\hline \multicolumn{2}{|l|}{ Schemes with Highest Fama Ratio } \\
\hline $\begin{array}{l}\text { Asset Management } \\
\text { Category }\end{array}$ & Schemes & $\begin{array}{l}\text { Fama } \\
\text { Ratio }\end{array}$ \\
\hline J. V. Indian & $\begin{array}{l}\text { ICICI Pru Exports and Other Services Fund - Dir } \\
\text { (D) }\end{array}$ & 0.07 \\
\hline J. V. Predominantly Indian & Reliance Small Cap Fund - Direct (D) & 0.07 \\
\hline Foreign AMC & $\begin{array}{l}\text { Franklin India Smaller Companies Fund - Direct } \\
\text { (D) }\end{array}$ & 0.08 \\
\hline J. V. Indian & SBI Small \& Midcap Fund - Direct (D) & 0.08 \\
\hline J. V. Indian & DSP BR Micro-Cap Fund - Direct (D) & 0.08 \\
\hline UTI & UTI-Transportation \& Logistics Fund - Direct (D) & 0.08 \\
\hline Indian Private AMC & $\begin{array}{l}\text { Motilal Oswal Most Focused Multicap 35 Fund- } \\
\text { Dir (D) }\end{array}$ & 0.08 \\
\hline Indian Private AMC & $\begin{array}{l}\text { Motilal Oswal Most Focused Midcap 30 Fund- } \\
\text { Dir (D) }\end{array}$ & 0.08 \\
\hline Indian Private AMC & Tata Resources \& Energy Fund - Direct (D) & 0.09 \\
\hline Indian Private AMC & $\begin{array}{l}\text { Tata Banking \& Financial Services Fund - Dir } \\
\text { (D) }\end{array}$ & 0.10 \\
\hline
\end{tabular}

Table 20

\begin{tabular}{|l|l|l|}
\hline \multicolumn{3}{|l}{ Schemes with Lowest Fama Ratio } \\
\hline $\begin{array}{l}\text { Asset Management } \\
\text { Category }\end{array}$ & Schemes & Fama Ratio \\
\hline J. V. Foreign & $\begin{array}{l}\text { Baroda Pioneer PSU Equity Fund - Plan B - } \\
\text { Dir (D) }\end{array}$ & -0.06 \\
\hline Indian Private AMC & $\begin{array}{l}\text { Tata India Pharma \& Healthcare Fund - Direct } \\
\text { (D) }\end{array}$ & -0.05 \\
\hline Indian Private AMC & $\begin{array}{l}\text { Sahara Banking \& Financial Services - Direct } \\
\text { (D) }\end{array}$ & -0.04 \\
\hline J.V. Indian & SBI PSU Fund - Direct (D) & -0.03 \\
\hline Indian Private AMC & Sundaram Growth Fund - Direct (D) & -0.02 \\
\hline Indian Private AMC & Tata Digital India Fund - Direct (D) & -0.02 \\
\hline Indian Private AMC & $\begin{array}{l}\text { Sundaram PSU Opportunities Fund - Direct } \\
\text { (D) }\end{array}$ & -0.02 \\
\hline Indian Private AMC & $\begin{array}{l}\text { Taurus Banking \& Financial Services - Direct } \\
\text { (D) }\end{array}$ & -0.02 \\
\hline Indian Private AMC & Indiabulls Value Discovery Fund - Direct (D) & -0.01 \\
\hline J. V. Indian & SBI Magnum Comma Fund - Direct (D) & -0.01 \\
\hline
\end{tabular}


Table 21

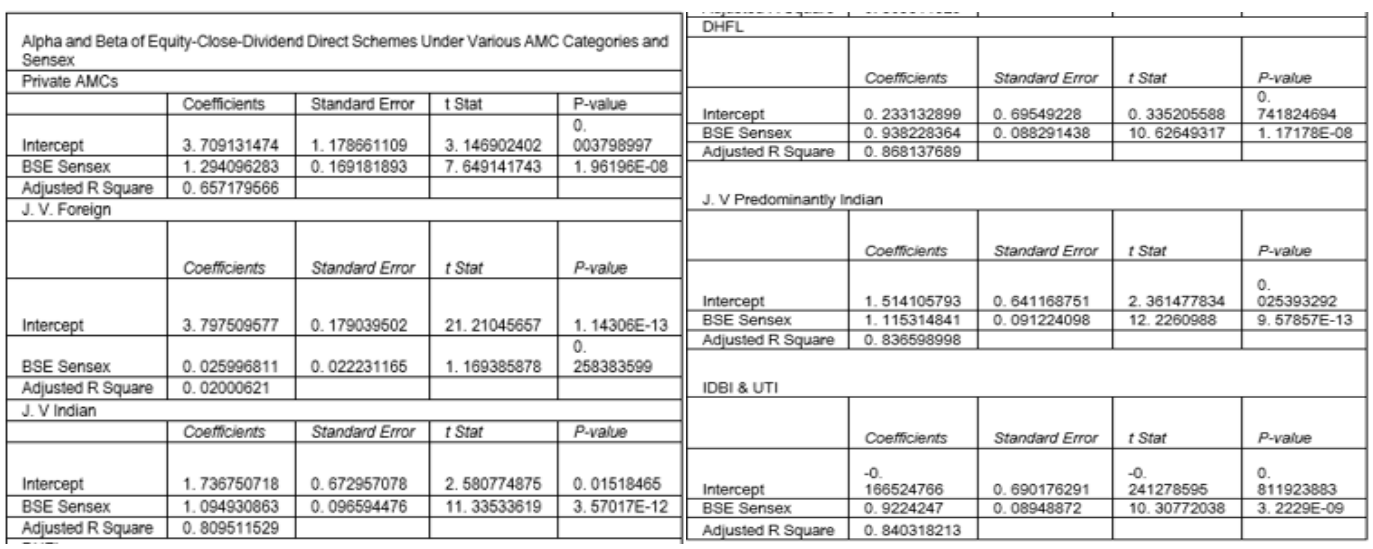

Table 22

\begin{tabular}{|c|c|c|c|c|c|c|c|}
\hline \multicolumn{8}{|c|}{$\begin{array}{l}\text { Correlation matrix of Equity-Close-Dividend Direct Schemes Under Various AMC Categories } \\
\text { and Sensex }\end{array}$} \\
\hline & $\begin{array}{l}\text { Private } \\
\text { AMCs }\end{array}$ & $\begin{array}{l}\text { J. } \quad V . \\
\text { Foreig } \\
n\end{array}$ & $\begin{array}{l}J . \quad V \\
\text { India } \\
n\end{array}$ & $\begin{array}{l}D H \\
F L\end{array}$ & $\begin{array}{l}J . \quad V \\
\text { predominantly } \\
\text { Indian }\end{array}$ & $\begin{array}{l}I D B I \\
\& \\
\text { UTI }\end{array}$ & $\begin{array}{l}\text { Sens } \\
\text { ex }\end{array}$ \\
\hline Private AMCs & 1.000 & & & & & & \\
\hline J. V. Foreign & 0.512 & 1.000 & & & & & \\
\hline J. V Indian & 0.953 & 0.451 & $\begin{array}{l}1 . \\
000\end{array}$ & & & & \\
\hline DHFL & 0.915 & 0.310 & $\begin{array}{ll}0 . \\
932\end{array}$ & $\begin{array}{l}1 . \\
000\end{array}$ & & & \\
\hline $\begin{array}{lr}J . & V \\
\text { predominantly } \\
\text { Indian }\end{array}$ & 0.937 & 0.439 & $\begin{array}{l}0 . \\
968\end{array}$ & $\begin{array}{l}0 . \\
942\end{array}$ & 1.000 & & \\
\hline IDBI \& UTI & 0.937 & 0.337 & $\begin{array}{l}0 . \\
974\end{array}$ & $\begin{array}{l}0 . \\
960\end{array}$ & 0.967 & $\begin{array}{ll}1 . \\
000\end{array}$ & \\
\hline Sensex & 0.818 & 0.273 & $\begin{array}{l}0 . \\
903\end{array}$ & $\begin{array}{l}0 . \\
936\end{array}$ & 0.918 & $\begin{array}{l}0 . \\
921\end{array}$ & $\begin{array}{l}1 . \\
000\end{array}$ \\
\hline
\end{tabular}

Table 23

\begin{tabular}{|c|c|c|c|c|c|c|}
\hline \multicolumn{7}{|c|}{$\begin{array}{l}\text { Shampe Ratio and Treynor Ralio of Equity-Close-Dividend Direct Schemes Under Various } \\
\text { AMC Cateonies }\end{array}$} \\
\hline $\begin{array}{l}\text { AMC } \\
\text { Cateleot }\end{array}$ & $\begin{array}{l}\text { Private } \\
\text { AMCS }\end{array}$ & $\begin{array}{l}\text { J. } V \text {. } \\
\text { Foveign }\end{array}$ & $\begin{array}{l}\text { J. V } \\
\text { Indian }\end{array}$ & DHFL & $\begin{array}{l}J . \quad V \\
\text { Predominanty } \\
\text { Indian }\end{array}$ & $\begin{array}{l}|D B| \& \& \\
U T \mid\end{array}$ \\
\hline lappe & $\begin{array}{l}\cdot 0 \\
548009 \\
306\end{array}$ & $\begin{array}{l}8 . \\
60050 \\
119\end{array}$ & $\begin{array}{l}0 \\
880476 \\
797\end{array}$ & $\begin{array}{l}-1 . \\
335553 \\
29\end{array}$ & -0.942717498 & $\begin{array}{l}-1 . \\
390867 \\
58\end{array}$ \\
\hline $\begin{array}{l}\text { yor } \\
\text { tio }\end{array}$ & $\begin{array}{l}2 . \\
784555 \\
066 \\
\end{array}$ & $\begin{array}{l}139 . \\
286314 \\
9\end{array}$ & \begin{tabular}{|l|}
4 \\
065096 \\
847 \\
\end{tabular} & $\begin{array}{l}6.6 \\
340673 \\
792 \\
\end{array}$ & -4.323442873 & $\begin{array}{l}6.6 \\
604333 \\
123 \\
\end{array}$ \\
\hline
\end{tabular}

